\title{
Conocimientos, actitudes y prácticas docentes en la educación para el emprendimiento*
}

\author{
Knowledge, attitudes and teaching practices in \\ education for entrepreneurship
}

Francia Milena Suárez García ** Janeth Chunga Hernández $\quad$ ***

\section{Resumen}

La labor docente no solo implica la formación, sino también la reflexión constante. Relacionado con la educación empresarial, desarrollar estudios que hagan posible esta revisión genera posibilidades para fortalecer y mejorar el proceso educativo. La investigación realizada en la institución educativa Fundación Universitaria Católica Lumen Gentium tiene como objetivo identificar los conocimientos, actitudes y prácticas de un grupo de docentes para establecer cómo se lleva a cabo esta educación empresarial. El acercamiento se realiza con un enfoque cualitativo de tipo descriptivo. Como principales resultados y conclusiones para enseñar el emprendimiento en esta institución se encontró que, en cuanto a los conocimientos, las asignaturas se desarrollan a través de un eje emprendedor desde el saber relacionado con la construcción y validación de un proyecto emprendedor; las actitudes respecto al emprendimiento de los docentes son positivas y favorables ya que se reconocen como emprendedores o les gustaría serlo y las prácticas confirman la orientación hacia el conocimiento; la mayoría de estrategias se llevan a cabo a través de procesos de instrucción, análisis y discusión, mayoritariamente dentro del aula.

Palabras clave: conocimientos, actitudes, prácticas, docentes, educación para el emprendimiento.

* Artículo producto de investigación.

* Psicóloga de la Universidad Cooperativa de Colombia, especialista en Docencia para la Educación Superior de la USC de Cali y magíster en Salud Pública de la Universidad del Valle. Docente investigadora en la Fundación Universitaria Católica Lumen Gentium. Correo electrónico: mae003@gmail.com; fmsuarez@unicatolica.edu.co; Orcid: 0000-0002-0192-7110

* Administradora de empresas de la Universidad del Valle, magíster en Administración de la Universidad Icesi y estudiante de Doctorado en Economía y Finanzas de la Universidad Benito Juárez. Docente investigadora en la Fundación Universitaria Católica Lumen Gentium. Correo electrónico: jchunga@unicatolica.edu.co; Orcid: 0000-0003-3721

* Administradora de empresas de la Universidad Autónoma de Occidente con maestría en Direccionamiento Estratégico de la Universidad del Mar de Cortés. Docente investigadora en la Fundación Universitaria Católica Lumen Gentium. Correo electrónico: mgonzalez@unicatolica.edu.co; Orcid: 0000-0003-2985-2891 


\section{Abstract:}

Teaching not only involves academic training, also constant reflection related to Business Education that developing studies that make this revision possible creates possibilities to strengthen and improve the educational process. The research conducted at the Fundación Universitaria Católica Lumen Gentium aims to identify knowledge, attitudes and practices of a group of teachers to establish how Business Education is being carried out. This approach uses a qualitative and descriptive approach. As main results and conclusions to teach entrepreneurship in this institution, it was found that, in terms of knowledge, the subjects are developed through an entrepreneurial axis from the knowledge related to the construction and validation of an entrepreneurial project; teachers' attitudes towards entrepreneurship are positive and favorable since they recognize themselves as entrepreneurs or would like to be so, and the practices confirm the orientation towards knowledge. Most strategies are carried out through instruction, analysis and discussion processes, mostly within the classroom.

Keywords: knowledge, attitudes, practices, teachers, education for entrepreneurship.

\section{Introducción}

El emprendimiento es un factor importante para el crecimiento económico y el progreso social de las naciones, por ello la enseñanza del emprendimiento constituye un eje primordial para el logro de estos objetivos. A través de la educación se brinda la posibilidad de involucrar la participación económica de las personas, impulsa el crecimiento y desarrollo de diferentes grupos sociales. En concordancia con la Organización para la Cooperación y el Desarrollo Económico (OCDE), "la educación y las competencias son factores decisivos para apoyar la transición de los jóvenes de la escuela al trabajo y el desarrollo incluyente", puesto que la educación es trascendental para "reducir la pobreza, eliminar desigualdades y construir estabilidad y cohesión social" (2016, p. 31).
La labor de los docentes es fundamental para generar y fortalecer estas competencias "incluyendo habilidades técnicas y blandas o transversales (creatividad, trabajo en equipo, compromiso, liderazgo, comunicación)" (OCDE, 2016, p. 16), para de esta manera, hacer posible un cambio en la sociedad hacia una cultura, como lo denomina Rodrigo Varela (2008), basada en un proceso educativo denominado educación empresarial. Los docentes no solo tienen que ver con la formación disciplinar relacionada con conocimientos y técnicas, sino con todo un conjunto de capacidades y habilidades que les permita a los educandos actuar y desenvolverse ante condiciones cada vez más cambiantes y exigentes; a la par, sus acciones deben estar atravesadas por procesos de revisión y reflexión constantes.

El presente estudio tiene como objetivo un acercamiento para identificar conocimientos, actitudes y prácticas de los docentes y así, analizar cómo se desarrolla la educación para el emprendimiento en una institución de educación superior. La educación para el emprendimiento comprende un proceso de creación de empresas y se relaciona con el concepto de educación empresarial que aborda Rodrigo Varela (2008; 2011).

En este documento se dan a conocer aportes de diferentes estudios relacionados con esta temática en Colombia, México, España, Alemania y Australia. Posteriormente, se adopta como marco de referencia la educación empresarial, se dan a conocer los elementos contextuales y metodológicos de la investigación y se presentan los hallazgos del estudio para confrontarlos con los antecedentes y marco de referencia. Por último, se esbozan conclusiones y se realizan recomendaciones producto del estudio.

\section{Antecedentes}

Relacionado con los conocimientos, actitudes y prácticas de los docentes de emprendimiento en instituciones educativas cabe mencionar los 
siguientes estudios. En 2011 Crissien dio a conocer los resultados de una investigación longitudinal realizada en la Universidad EAN (Escuela de Administración de Negocios, Colombia) en la cual se llevaron a cabo mediciones en 2008 y 2010 a estudiantes y egresados sobre la incidencia de la formación empresarial en la deseabilidad de ser empresarios. Como resultado destacado se puede mencionar que antes de iniciar el programa emprendedor de esta institución, el $65 \%$ de los estudiantes declararon querer ser empresarios, luego 92 \% declararó querer ser empresario al culminar el programa emprendedor.

En sus deliberaciones, este mismo autor, da a conocer la manera en la que el papel del docente en la enseñanza del emprendimiento debe estar mediado por la reflexión y actualización de las estrategias pedagógicas, así como el reconocimiento de la individualidad y pluralidad de los educandos y su entorno, ya que estos son primordiales para poder llevar a cabalidad la formación de empresarios (2011).

Existen posibilidades metodológicas desconocidas por muchos profesores y este desconocimiento genera una visión de túnel al pensar que solo ciertos conocimientos deben ser trasmitidos al educar y formar potenciales empresarios [...] el aprendizaje es individual y surge, de la autonomía de los estudiantes frente a un proyecto de vida y las necesidades para lograrlo [...] el aprendizaje libera, forma, transforma individuos con un sistema de creencias dado, en seres más desarrollados para un bien mayor. (Crissien, 2011, p. 108)

Adicionalmente, este estudio revela que la educación empresarial se orienta a la formación para la creación de empresas, este proceso no solo debe contemplar actividades en el aula de clase. Desde su perspectiva, las estrategias pedagógicas que se desarrollan por fuera del salón como visitas a empresas, entrevistas a empresarios en su entorno, así como otras relacionadas con el aprendizaje experiencial, tales como ferias y concursos de planes de negocio son idóneas para la formación de empresarios (Crissien, 2011).

La investigación "Línea base de conocimiento de las unidades de emprendimiento en las instituciones de educación superior en Colombia" efectuó una revisión documental y una encuesta semiestructurada a 55 responsables de emprendimiento de instituciones de educación superior de la Red Universitaria de Emprendimiento (Reune) dio a conocer que en estas instituciones el acceso al conocimiento del emprendimiento se desarrolla a través de estos tres tipos de cátedras: disciplinar, electiva y transversal, un $49 \%$ corresponde a cátedras disciplinares, el $35 \%$ corresponde a cátedras electivas y el $16 \%$ a cátedras transversales. (Cubillos y Rodríguez, 2013)

Las instituciones de educación superior ejecutan otro tipo de actividades diferentes a las incluidas en las estructuras curriculares, se identifican entre ellas: conversatorios y charlas en un $20 \%$; conferencias y foros en $17 \%$; talleres y juegos de roles en 14 $\%$; muestras, ferias o concursos en un $13 \%$; presentación de proyectos y planes de negocio en un 12 $\%$; simposios y encuentros con expertos en un $11 \%$; visitas y salidas de campo en $8 \%$; lo cual deja en último lugar las ruedas de negocios con $6 \%$. Se observa que el uso de las Tecnologías de la Información y las Comunicaciones (TIC) es bajo y corresponde a un $14 \%$, contra un $86 \%$ de las demás actividades. El mayor uso de estas herramientas se da a través de aulas virtuales y blogs. El uso de simuladores también es bajo con un 22 \% (Cubillos y Rodríguez, 2013, pp. 304-305). Las anteriores investigaciones se centran en el estudio de las prácticas pedagógicas utilizadas en instituciones de Colombia.

En este mismo sentido, en el ámbito internacional, la institución de educación superior Centro de Diseño, Cine y Televisión (Centro) en México desarrolla un estudio piloto con estudiantes de último año de sus licenciaturas. En este, deben cursar una asignatura de 
emprendimiento. El objetivo de esta asignatura tiene como fin que los educandos diseñen un proyecto emprendedor en el ámbito de la economía creativa.

En el estudio piloto se consideraron resultados de investigaciones que dan cuenta de que la enseñanza del emprendimiento es más efectiva cuando se realizan procesos de aprendizaje experiencial en grupos pequeños e interdisciplinarios en los cuales se privilegian ambientes creativos y colaborativos, acompañados de actividades extracurriculares que ayuden a la inspiración y ejecución del proyecto a través de siete módulos, los cuales abordan temas que incluyen: segmentación de clientes y propuesta de valor, etnografía, diseño centrado en usuarios en el módulo 1; gestión de talento y conformación de equipos en el módulo 2; canales y relaciones con los clientes en el módulo 3; manejo de recursos y alianzas en el módulo 4; revisión de los temas de ingresos y costos en el módulo 5; práctica con base en los prototipos en el módulo 6; y presentación de proyectos y retroalimentación de jueces externos en el módulo 7 (Traverso y Paniagua, 2015).

Sobre las prácticas pedagógicas implementadas por los docentes en esta institución se puede afirmar que de los módulos 1 a 6 se trabajó de forma presencial, con un formato de clase que incluía exposición del profesor, análisis de casos, retroalimentación de los alumnos y síntesis de los hallazgos. El módulo 7 se realizó con un formato de panel conformado por jueces invitados. Todo el proceso se acompañó con actividades extramuros, de acuerdo con las necesidades de cada equipo: visitas de campo, entrevistas con expertos, visitas de inspiración, entrevistas en empresas y medios de comunicación (Traverso y Paniagua, 2015, p. 9).

Los docentes que impartieron las sesiones desarrollaron exposiciones de contenido, seguidas de preguntas y análisis de ejemplos. En estos espacios también se presentaron exposiciones de avances de los alumnos con retroalimentación de mentores e invitados, y atención de consultas de los alumnos. Cabe destacar que los docentes de este proceso también apelaron a la empatía para atender a situaciones de frustración que presentaron los estudiantes. Todos los estudiantes participantes formularon ideas coherentes y presentaron un prototipo para el desarrollo de un negocio en el ámbito de la economía creativa con diferentes niveles de viabilidad.

Traverso y Paniagua (2015) determinaron que el estudio piloto fue útil para el mejoramiento de las técnicas en posteriores ediciones del curso de la institución, así mismo, dieron a conocer que es factible enseñar emprendimiento con base en el método de caso y aprendizaje experiencial.

Teniendo en cuenta el impacto que tiene la enseñanza de los educadores en los estudiantes y en la sociedad, Robinson, Neergaard, Tanggaard y Krueger (2016) realizaron un estudio etnográfico sobre el desarrollo continuo y la implementación de un curso en un periodo de diez años. La intención del curso fue desarrollar la mentalidad emprendedora en los estudiantes, el cual busca alejarse del paradigma de la educación universitaria tradicional, aquella de graduar empleados y no emprendedores. Para hacerlo posible, incorporaron cuatro diferentes perspectivas teóricas para la enseñanza del emprendimiento: 1) conductismo; 2) aprendizaje social de Bandura; 3) aprendizaje situado -que tiene en cuenta el contexto sociocultural-; y 4) procesos de aprendizaje existencial.

Dentro de sus hallazgos plantean que la combinación de modelos teóricos parece mejorar el aprendizaje debido a que las personas tienen estilos diferentes de aprendizaje. Para lograr que los estudiantes desarrollen una mentalidad emprendedora los autores sostienen que los docentes se deben alejar de la educación dirigida por el maestro y encaminarse hacia unas prácticas más centradas en el alumno, ya que necesitan involucrar a los estudiantes como co-creadores de la clase a fin de promover el empoderamiento en el proceso de aprendizaje. 
En la investigación sobre el papel del profesor en la educación emprendedora en España, Núñez y Núñez (2018) plantean que la educación emprendedora es un "proceso mental, una forma de aprender, un tipo de pedagogía, dirigida a desarrollar las habilidades y competencias del individuo emprendedor" (p. 31), de acuerdo con estos autores, el docente y su didáctica son los principales agentes para desarrollar y fortalecer dichas competencias. Este estudio se llevó a cabo con 175 docentes de educación primaria y secundaria.

En sus hallazgos dan cuenta de que la actitud positiva de los profesores hacia la educación emprendedora está influida por su propia intención de emprender. Entre otros aspectos que afectan positivamente la orientación de acciones hacia el emprendimiento están la asignatura que imparten y la edad de los docentes. Los docentes que dictan asignaturas relacionadas con temáticas empresariales y los docentes menores de 35 años son los más proclives a su puesta en práctica.

Acerca de otros enfoques y métodos de enseñanza - aprendizaje para el emprendimiento en educación superior en el mundo, cabe mencionar el estudio de caso de Bliemel en la Universidad de Nueva Gales del Sur, Australia (2014) que da cuenta de cómo su programa con cursos básicos centrados en los fundamentos del emprendimiento, liderazgo y comunicación, basados en el aprendizaje experiencial con enfoque gradual, facilita a los estudiantes el proceso de actuar y pensar como verdaderos empresarios. Los tres niveles del programa son:

1. Experiencias indirectas a través de oradores invitados y mentores

2. Experiencias virtuales a través de presentaciones en el aula para inversores reales

3. Experiencias directas (opcionales) a través de la presentación de sus ideas en eventos de redes de la industria y concursos de presentación
Las prácticas académicas están encaminadas a que los estudiantes salgan de su zona de comodidad, fuera del aula de clase. Otro aspecto destacado de este estudio se relaciona con las reflexiones de Bliemel, partidario de la heterogeneidad de los programas de educación empresarial que tenga en cuenta los diferentes contextos; más allá de la dificultad de evaluarlos y compararlos, para Bliemel la diversidad de programas representa una oportunidad para aprender de cada uno.

Como resultados del programa se documenta que tiene una buena recepción en los estudiantes e incrementa su interés y compromiso para convertirse en empresarios. Referente a la evaluación de la enseñanza, el curso recibió una calificación constante de 4 a 10 puntos, más alto que el promedio de otros cursos de las facultades. De igual forma, en un periodo de dos años (2011 a 2013), algunos estudiantes del programa de enseñanza del emprendimiento que participaron en competiciones obtuvieron los primeros puestos en siete competencias nacionales, tres premios para comercialización y lanzamiento de productos, y a nivel internacional, el desarrollo de una Startup en Silicon Valley.

Mindt y Rieckmann (2017) realizaron un análisis de 183 publicaciones científicas relacionadas con el desarrollo de competencias para el emprendimiento, los enfoques de enseñanza-aprendizaje descritos en estos artículos fueron: aprendizaje colaborativo con 19,4 \%; aprendizaje experimental con 18,9 \%; aprendizaje basado en problemas con 7,6 \%; aprendizaje centrado en el alumno con 7,2 \%; aprendizaje con base en proyectos 7,1 \%; aprendizaje transformativo con 6,1 \%; aprendizaje en el mundo real con 4,6 \%; aprendizaje reflexivo con 4,3\%; aprendizaje basado en el contexto con 4,1\%; aprendizaje activo con 3,6 \%; coaching, mentoring y modelling con 3,1 \%; otras modalidades con $14,1 \%$, las cuales combinan aprendizaje interdisciplinar, tradicional, virtual, creativo, entre otros. 
En este caso, se observa que los autores refieren con mayor frecuencia el aprendizaje colaborativo y experiencial frente al tradicional, el cual alcanzó solo el 2,6\%. Este resultado proporciona información sobre las prácticas de enseñanza y aprendizaje que son tendencia a nivel mundial. Se identifica un amplio abanico de posibilidades que se podría explorar en determinados casos y que podrían representar opciones adaptadas al contexto, como insumo para el diseño y modificación de cursos y programas de emprendimiento. Estos autores también afirman que encontraron que pocos autores se refieren a las políticas publicadas por organizaciones nacionales e internacionales (Mindt y Rieckmann, 2017).

\section{Marco de referencia}

\section{La educación empresarial planteada por Rodrigo Varela}

En el presente estudio se tienen en cuenta los aportes de Varela, quien posee una amplia trayectoria académica y se le reconoce como un estudioso de la educación empresarial. Su modelo tiene en cuenta experiencias y elementos teóricos y prácticos de otros modelos que han sido exitosos, incorpora las competencias que otorgan relevancia a otras dimensiones muy importantes del ser humano, y fuera de ello, es una propuesta flexible y adaptable a las necesidades culturales de otras poblaciones.

Rodrigo Varela (2011), afirma que la educación empresarial permite un proceso de transformación y desarrollo económico en el cual el sistema educativo ha de generar cambios al reevaluar el modelo de educación tradicional o "empleomanial" por otro en el cual se tenga en cuenta el marco contextual específico. Los principales elementos que recoge el modelo de Varela se presentan en la tabla 1, la cual permite evidenciar que la labor docente se debe centrar en el estudiante y sus competencias personales y profesionales para ayudar a la construcción de los proyectos de vida de los educandos.

Tabla 1. Sistematización y análisis del modelo propuesto por Rodrigo Varela

Objetivo: la educación empresarial se enfoca en crear una cultura empresarial para promover la aparición de empresarios capaces de crear empresas competitivas.

\begin{tabular}{|c|c|c|c|}
\hline Caracteristicas & $\begin{array}{l}\text { Conocimientos y } \\
\text { competencias }\end{array}$ & Recursos & Ejercicios pedagógicos \\
\hline $\begin{array}{l}\text { Está centrado en el } \\
\text { estudiante. Es flexible. } \\
\text { Propende por el desarrollo } \\
\text { de competencias concep- } \\
\text { tuales y personales. Se } \\
\text { aleja del modelo educativo } \\
\text { "tradicional". Busca generar } \\
\text { cambio de actitudes. } \\
\text { Involucra un trabajo } \\
\text { integral desde distintos } \\
\text { componentes. Generación } \\
\text { de interacción de grupos y } \\
\text { pensamiento divergente. }\end{array}$ & $\begin{array}{l}\text { Espíritu empresarial; } \\
\text { Proceso empresarial; } \\
\text { Técnicas de evaluación de } \\
\text { oportunidades; mercadeo } \\
\text { de nuevas empresas; } \\
\text { comercialización de } \\
\text { innovaciones; liderazgo; } \\
\text { creatividad e innovación; } \\
\text { plan de empresa; } \\
\text { planeación financiera } \\
\text { para una nueva empresa; } \\
\text { administración de } \\
\text { empresas en crecimiento; } \\
\text { valoración de empresas } \\
\text { en crecimiento; estrategia } \\
\text { empresarial y mecanismos } \\
\text { de apoyo, entre otros. }\end{array}$ & $\begin{array}{l}\text { Formadores; asesores; } \\
\text { mentores; escenarios de } \\
\text { prácticas empresariales; } \\
\text { Software de plan de em- } \\
\text { presa; concursos de plan de } \\
\text { empresa; ferias empresaria- } \\
\text { les; contactos con organis- } \\
\text { mos de financiación; formas } \\
\text { de incubación de empresa; } \\
\text { ambientes de aprendizaje } \\
\text { ajustados a las necesidades } \\
\text { de los estudiantes. }\end{array}$ & $\begin{array}{l}\text { Estimulación de las capacidades } \\
\text { creativas e innovadoras a través de } \\
\text { juego de roles, teatro, dibujos, etc.; } \\
\text { actividades empresariales reales: } \\
\text { búsqueda de ideas y de empresa- } \\
\text { rios; desarrollo de investigaciones; } \\
\text { creación de presentaciones en } \\
\text { las que la clase dependa en gran } \\
\text { medida de las acciones e iniciativas } \\
\text { del estudiante; actividades para el } \\
\text { desarrollo de valores éticos para la } \\
\text { toma de posiciones personales dando } \\
\text { importancia a la conducta honesta } \\
\text { e íntegra en el mundo empresarial. }\end{array}$ \\
\hline
\end{tabular}

Fuente: elaboración propia con base en Varela (2011) 
Por otro lado, este modelo también busca reforzar las relaciones interpersonales de los estudiantes a través de la interacción con grupos empresariales universitarios, clubes de empresarios, redes de empresarios, ferias, exposiciones y congresos como elementos facilitadores del proceso formativo empresarial.

Para Varela el reto del sistema educativo y de los educadores consiste en formar empresarios capacitados mediante ejercicios pedagógicos que les permitan actuar en condiciones ambientales exigentes, que desarrollen capacidad de logro y realización, corran riesgos moderados, definan y aprovechen oportunidades, a su vez, que estén interesados en aprender, actualizarse y mejorar, con lo cual se crean fuentes, no solo de empleo sino también de bienestar, en un marco ético y de responsabilidad social. Adicionalmente, Varela también considera que la preparación del estudiante universitario para el mundo empresarial debe evitar el paradigma de la educación tradicional, el cual trabaja con verdades absolutas, decisiones basadas en hechos cuantificables y determinísticos, situaciones de tipo binario, presencia pasiva en el salón de clase y evitación de riesgos (Varela, 2008).

\section{Descripción del estudio de caso}

El estudio se desarrolló en la Fundación Universitaria Católica Lumen Gentium en la ciudad de Cali, Colombia. Esta universidad se divide en facultades y programas, cuenta con cinco facultades, doce programas de pregrado, cuatro programas de tecnología y un programa técnico. Inicialmente, en la Facultad de Ciencias Empresariales se creó el Programa de Emprendimiento, posteriormente, la universidad adoptó esta iniciativa y brinda educación emprendedora a toda la comunidad académica en sus diferentes facultades y programas.

Dentro de la Facultad de Ciencias Empresariales se encuentra el Programa de Administración de Empresas. A diferencia de los demás programas en los cuales solo se dictan una o dos asignaturas de emprendimiento, esta carrera sitúa su énfasis en el emprendimiento y en el currículo se desarrolla el eje de emprendimiento con asignaturas como: espíritu emprendedor; plan emprendedor l; plan emprendedor II; plan emprendedor III; plan emprendedor IV; electiva en innovación; electiva en emprendimiento; gerencia de proyectos; práctica empresarial; mercadeo; cooperación internacional; y mercadeo internacional.

Estas asignaturas son obligatorias para todos los alumnos del programa y al final se espera que los estudiantes se gradúen, previa presentación y sustentación de un proyecto emprendedor, sin embargo, existen otras alternativas de modalidad de trabajo de grado para los alumnos que no opten por la presentación del proyecto emprendedor, como por ejemplo: desarrollo de trabajo de gestión empresarial, desarrollo de trabajo de investigación o pasantía internacional.

\section{Metodología}

El estudio se desarrolla con un enfoque cualitativo de tipo descriptivo. De acuerdo con Quintana (2006), Mejía (2000) y Pizarro (2000), este enfoque permite captar planos de la realidad humana a través de la lógica y el sentir de sus protagonistas, como, por ejemplo, la significación de las acciones humanas cotidianas, que en este caso en particular se agruparon en un conjunto los conocimientos, actitudes y prácticas relacionados con el quehacer habitual de un grupo de docentes.

Se empleó el análisis de contenido puesto que, de acuerdo con López, esta técnica "se sitúa en el ámbito de la investigación descriptiva, pretende, sobre todo, descubrir los componentes básicos de un fenómeno determinado extrayéndolos de un contenido dado" (2002, p. 174). Para este caso solo se adopta la vertiente cualitativa que valida Cáceres (2003). 
El análisis cualitativo de contenido se define a sí mismo dentro de este marco de trabajo como una aproximación empírica, de análisis metodológicamente controlado de textos al interior de sus contextos de comunicación, siguiendo reglas analíticas de contenido y modelos paso a paso, sin cuantificación de por medio.

El proceso metodológico se desarrolla con la recopilación de información y su sistematización, de acuerdo con los detalles especificados en la tabla 2 .

Tabla 2. Procedimiento de análisis cualitativo del contenido utilizado

\section{Captura}

Aplicación de un instrumento (cuestionario) con preguntas abiertas al grupo de docentes que dictan las asignaturas del eje de emprendimiento de la Facultad de Ciencias Empresariales detalladas en la descripción del caso. Respondieron 13 de los 16 docentes.

El instrumento indaga sobre los conocimientos de conceptos de emprendimiento, conocimientos impartidos en las asignaturas dictadas, actitudes hacia el emprendimiento de los docentes y prácticas relacionadas con las estrategias de enseñanza.

\section{Sistematización}

- Técnica de análisis de contenido,

- Organización de los resultados del cuestionario en hojas de Excel que incluyen preguntas y respuestas de manera fiel a los cuestionarios diligenciados por los docentes.

- A partir de los resultados del instrumento se hizo una definición inicial de las unidades de análisis.

- Establecimiento de reglas de análisis;

- Desarrollo de la codificación;

- Estructuración de categorías: conocimientos, actitudes y prácticas.

Fuente: elaboración propia

\section{Resultados, análisis y discusión}

\section{Conocimiento de los docentes}

A partir de esta categoría, gracias a las respuestas de los docentes se recaba información sobre el significado de los términos emprendimiento e innovación, las teorías, metodologías y modelos que conocen y utilizan, sus consideraciones sobre las asignaturas y los contenidos que se deberían dictar.

Sobre el significado del término emprendimiento, la mayoría de los docentes ${ }^{1}$ lo definen con emprender una idea o negocio, sin embargo, algunos de ellos complementan esa definición al incluir la importancia de realizar un buen análisis de mercado.

Es una idea de negocio materializada con el esfuerzo y dedicación del emprendedor, dicha idea es producto de un análisis visionario del mercado. (respuestas al cuestionario del docente 3)

Es toda actitud que tengan las personas para proponer nuevas formas de realizar procesos o nuevos productos y servicios que ayuden a suplir una necesidad de cualquier grupo social. (respuestas al cuestionario del docente 9)

Como se puede apreciar, en la declaración del docente 9 también se relaciona el emprendimiento con una actitud que no solo ayuda a satisfacer necesidades particulares sino de una sociedad.

Frente al concepto de innovación los docentes enfocan la definición hacia diferentes procesos o áreas. Algunos relacionan el concepto con crear o realizar ideas originales, diferentes a lo que ya existe. Sin embargo, lo complementan con la importancia que tiene que el mercado acepte estas ideas y las reconozca. Para ellos la innovación es aplicable a productos, servicios e individuos, agrega valor y construye tejido social.

$\overline{1}$ En este caso se utiliza este término como complemento de la descripción con el fin de hacer afirmaciones acerca de los datos 
Cuando se indaga sobre las teorías que utilizan para apoyar el proceso de enseñanza mencionan teorías, modelos y metodologías de emprendimiento y administración, estas se especifican en la tabla 3.

Tabla 3. Descripción de teorías, metodologías y modelos para la enseñanza del emprendimiento de los docentes de Unicatólica en 2017

\begin{tabular}{l|l|l}
\hline Teorías & Metodologías & Modelos \\
\hline Teoría numérica & Prueba Gamba; & Diamante \\
en énfasis; teoría & estudio de & competitivo; \\
clásica de la & mercado; justo a & ciclo PHVA \\
administración; & tiempo; modelo & \\
teoría neoclásica; & de Gibb; modelo & \\
teoría de & de Shapero; & \\
sistemas; teoría & innovación & \\
de la motivación; & sistemática & \\
teoría de & & \\
Schumpeter; & & \\
teoría de & & \\
Stevenson; & & \\
teoría de & & \\
Mc Clelland; & & \\
teoría de Freire & & \\
\hline
\end{tabular}

Fuente: elaboración propia

Con los resultados de esta tabla se puede apreciar que en Unicatólica no se aplica un modelo institucional, en su lugar, los docentes adoptan gran diversidad de opciones para educar sobre emprendimiento, cada profesor aplica la teoría, metodología y/o modelo que considera más apropiados. Si se tiene en cuenta lo expuesto por Bliemel (2014), la diversidad se puede convertir en oportunidad para aprender de los distintos desarrollos.

En relación con las múltiples asignaturas en emprendimiento que posee la malla curricular, los docentes mencionan que faltan asignaturas sobre innovación y creatividad, modelos de negocios como emprendimiento naranja, negocios ecológicos y tecnológicos.

Faltan asignaturas sobre innovación y creatividad. (respuestas al cuestionario del docente 4) Falta una asignatura que involucre trabajo de campo e investigación en emprendimientos naranjas, ecológicos, tecnológicos y empresariales. (respuestas al cuestionario del docente 10)
Un docente establece que no faltan asignaturas dentro de la malla curricular y que lo que se necesita es que los docentes del eje emprendedor conozcan la malla y se direccionen las asignaturas existentes.

No conozco en detalle toda la malla, pero considero que el problema no está en tantas asignaturas si no en direccionar las existentes. (respuestas al cuestionario del docente 12)

Las declaraciones de los docentes afirman que la educación emprendedora en la institución se debería basar en la práctica, como lo propone Varela al privilegiar la educación centrada en el estudiante.

La enseñanza debería tener mayor énfasis en la práctica, no solo realizar un plan de negocios en el papel. (respuestas al cuestionario del docente 5)

La enseñanza del emprendimiento debería ser mediante el uso de metodologías activas, donde el estudiante participe activamente en el proceso de aprendizaje al usar estrategias didácticas novedosas y creativas. (respuestas al cuestionario del docente 8)

Las asignaturas deberían contener las visitas de emprendedores que puedan contar cada experiencia, pero estas visitas deben ser subsidiadas por la institución y así garantizar la presencia de una persona externa una vez por semestre en cada asignatura. (respuestas al cuestionario del docente 9)

Los docentes desarrollan los contenidos de sus asignaturas a través de diversas teorías y modelos, se contempla el seguimiento de micro currículos proporcionados por la institución, pero no se habla del seguimiento de un modelo institucional.

Como respuesta a la pregunta ¿qué considera que se debería enseñar?, resaltan las siguientes respuestas: 
Se deben enseñar los temas establecidos en el micro currículo, considero que son determinantes. (respuestas al cuestionario del docente 7)

Considero que el micro currículo entregado por la universidad para su enseñanza está acorde con lo que se debe enseñar en la asignatura. (respuestas al cuestionario del docente 11)

Como respuesta a la pregunta ¿Cuáles teorías maneja en su asignatura del eje emprendedor?, resaltan las siguientes respuestas:

Ciclo PHVA, Diagrama causa-efecto, justo a tiempo, lean manufacturing, control de calidad, cadena de suministros. (respuestas al cuestionario del docente 7)

Teoría de Freire, donde todo proceso emprendedor combina tres componentes: ideal, capital y emprendedor. Teoría de Stevenson, donde el emprendimiento es un sistema de gestión que se debe apoyar en personas emprendedoras llamadas líderes comerciales. (respuestas al cuestionario del docente 11)

Los contenidos del eje emprendedor de Unicatólica manejan similitudes con los de otras instituciones y propuestas expuestas por Varela (2011), Traverso y Paniagua (2015), ya que incorporan el espíritu emprendedor, estudios de mercado, infraestructura del proyecto, proceso empresarial, viabilidad económica e innovación; algunos de los docentes coinciden al manifestar la necesidad de fortalecer en el eje emprendedor las competencias relacionadas con la creatividad.

Pero, al tener en cuenta los hallazgos y consideraciones de autores como Varela (2011), Bliemel (2014), Núñez y Núñez (2018) y Crissien (2011), para quienes la educación emprendedora es un proceso integral al cual se deben incorporar otras competencias relacionadas con el ser, se encuentran posturas diferentes.

Referente a lo destacado por Mindt y Rieckmann (2017), para quienes el desarrollo de competencias individuales debe apuntar a la creación de entornos de aprendizaje en los que se puedan desarrollar competencias que se dirijan al surgimiento de empresas innovadoras orientadas a la sostenibilidad en Unicatólica, no se encontró énfasis.

\section{Actitudes de los docentes hacia el emprendimiento}

En este aspecto se exploran las creencias, percepciones e intenciones. Se identifica la creencia de que el emprendimiento es algo positivo y se observa entre los docentes una predisposición hacia este; tienen una valoración favorable del emprendimiento, puesto que lo califican como importante y valioso.

Sí, soy emprendedora, con un sueño que desde la universidad se gestó con un pequeño de grupo de compañeros(as), se creó una empresa de asesorías integrales para los diferentes proyectos.

La experiencia ha sido enriquecedora, ya que desde la universidad se pensó [el proyecto] y hoy en día se ha puesto en marcha. Sí ha tenido sus inconvenientes, pero estos específicamente se dieron al inicio del proyecto, cuando de penetrar el mercado se trató, de ahí la importancia de educar al estudiante en la lucha por mantener viva y hacer realidad su idea de negocio y/o emprendimiento. (respuestas al cuestionario del docente 11)

Los docentes se reconocen -en gran parte- como emprendedores, los que no han sido emprendedores les gustaría serlo, los que sí lo son quieren continuar y a los que lo fueron anteriormente les gustaría volver a serlo. 
La actitud favorable hacia el emprendimiento se refleja en conductas que buscan motivar a los estudiantes, tales como:

- Estimular la capacidad de soñar (respuestas al cuestionario del docente 1).

- Inspirar a través del ejemplo (respuestas al cuestionario del docentes 4 y 11).

- Inspirar a través de la identificación con otros con las historias de personajes significativos y experiencias exitosas. (respuestas al cuestionario de los docentes 2 y 3 ).

- Promocionar los beneficios del emprendimiento, entre estos presentar el emprendimiento como una opción a la búsqueda de empleo para generar ingresos (respuestas al cuestionario de los docentes, 6, 9 y 13).

- El emprendimiento se valora como una experiencia enriquecedora, trascendental, que solo con su práctica en el "mundo real" se transforma en el elemento principal para la construcción de un saber, también, se considera importante motivar la constancia de los estudiantes hacia el "esfuerzo y la perseverancia" (respuestas al cuestionario de los docentes 1 y 10 ).

Los profesores perciben que el emprendimiento "no es una tarea fácil", puesto que la realidad plantea retos, inconvenientes y obstáculos que desafían las competencias del emprendedor. Dentro de los principales inconvenientes para los que se debe preparar a los educandos, los docentes que han sido emprendedores destacaron los siguientes:

- Fallas en la estructuración del mercado (respuestas al cuestionario de los docentes $1-3,5,10$ y 11).
- Desequilibrios en la vida personal y familiar (respuestas al cuestionario de los docentes 8 y 6 ).

- Diferencias entre socios (respuestas al cuestionario de los docentes 9 y 10)

- Fallas en el análisis del entorno (respuestas al cuestionario de los docentes 6, 12 y 13)

- Falta de tiempo para administrar el negocio de forma directa y permanente (respuestas al cuestionario del docente 5 ).

Los sentimientos no placenteros que dichas experiencias pudieron haber generado, no disminuyeron significativamente la valoración favorable de este conjunto de docentes con respecto al emprendimiento. Las actitudes favorables de los docentes se expresan en su interés por permanecer actualizados en el tema, concurrir a seminarios y capacitaciones en los cuales participan como promotores, asistentes, expositores, y en algunos casos como organizadores de ferias y workshops o talleres de emprendimiento, así como investigar y generar trabajos de campo con los estudiantes.

Sí, participo de ferias - workshops. (respuestas al cuestionario del docente 5)

Yo participo de seminarios y capacitaciones. (respuestas al cuestionario del docente 6)

Desarrollo capacitaciones, seminarios, ferias empresariales en Unicatólica cuando me asignan los grupos de emprendimiento. Desarrollo la investigación en el aula para crear cultura en emprendimiento y realizo con los estudiantes trabajo de campo en la investigación de mercados. (respuestas al cuestionario del docente 10) 
Los docentes manifestaron una actitud positiva hacia el emprendimiento. De acuerdo con el estudio español de Núñez y Núñez (2018), su actitud positiva puede estar influenciada por sus experiencias y propia intención de emprender.

Para la institución, la predisposición y valoración de los docentes con respecto al emprendimiento, - visto como importante- se puede convertir en un factor facilitador para el desarrollo del eje emprendedor. Las intenciones por actualizarse en el tema, capacitar, promover e investigar, sumado al reconocimiento del emprendimiento como valioso le resulta favorable para generar y consolidar el compromiso de este recurso humano en los proyectos que plantee la institución en esta línea.

\section{Prácticas en la educación emprendedora}

Como estrategias de enseñanza desarrolladas por los docentes en las asignaturas de emprendimiento, algunos adoptan la clase magistral, que se combina con actividades participativas en el aula para facilitar la interacción con los estudiantes. Emplean herramientas audiovisuales, análisis de lecturas, estudio de casos, búsquedas bibliográficas dirigidas, sondeo de conocimientos previos y de las expectativas de los alumnos. Asimismo, mencionan que adoptan instrumentos en clase como las presentaciones y el taller, también comentaron emplear el modelo de negocio Canvas, una herramienta de dirección estratégica y emprendimiento que permite describir, diseñar, desafiar, inventar y pivotar modelos de negocio (Strategyzer, 2020). En cuanto al uso de software un solo docente emplea el simulador de negocios que adquirió la universidad y otro usa la prueba Gamba, es una prueba de opción múltiple, cuyos resultados a nivel orientativo permiten identificar las habilidades emprendedoras de una persona.

En menor medida, un docente opta por el uso de las visitas empresariales, otro maneja las tutorías personalizadas; una docente utiliza la entrevista a emprendedores para configurar casos de estudio en clase y otra utiliza la sustentación de las ideas de negocio de los estudiantes en el espacio de clase con retroalimentación de los compañeros, profesores invitados y docente encargado, práctica similar a la que presentan Traverso y Paniagua (2015) y Bliemel (2014).

Uno de los docentes participantes expone como condición destacada para la enseñanza del emprendimiento que el alumno exprese o refleje su motivación de emprender, idea que Varela (2008; 2011) tiene en cuenta en su modelo y lo considera como un aspecto del proceso de enseñanza del emprendimiento.

Según lo expresado, existe interés de los profesores por conseguir la participación del estudiante en la clase. Una porción de ellos también tiene en cuenta las motivaciones para el emprendimiento, experiencias y conocimientos previos de los estudiantes, como lo mencionan Varela (2011), Crissien (2011) y Bliemel (2014). Otros docentes se enfocan en la comparación de la experiencia de otros versus el conocimiento.

Los espacios que adopta el profesorado para la enseñanza de la asignatura son en su mayoría espacios institucionales: aula de clase física y aula de clase virtual; en un caso se reporta el uso de la biblioteca de la universidad y en otros dos, el uso de espacios externos para las visitas empresariales y la entrevista a un emprendedor. Este hallazgo es contrario a lo que recomiendan autores como Crissien (2011), Traverso y Paniagua (2015), Bliemel (2014) y Varela (2011).

Los docentes consideran que el resultado de este proceso de enseñanza se debe reflejar en una idea de negocio medida y acompañada desde su nacimiento hasta su ejecución. Actualmente, en la institución el proceso llega hasta la presentación del proyecto de emprendimiento.

Según el modelo de Varela (2011), el proceso de educación empresarial debe disponer de recursos, entre ellos software. Llama la atención que en Unicatólica la 
institución posee un simulador pero solo un docente lo utiliza y otro docente emplea otro recurso digital, esto coincide con el estudio de Cubillos y Rodríguez (2013) en el cual, en primer lugar, el uso de TIC es bajo y dentro de los recursos utilizados, las aulas virtuales también son la herramienta de mayor aplicación.

En esta institución los docentes adoptan una combinación diversa de estrategias, el conocimiento sobre emprendimiento que se imparte a los alumnos se genera, en gran medida, a través de procesos de instrucción, análisis y discusión dentro del aula. Contrario a lo reportado en la literatura en el desarrollo de las asignaturas, se tienen en cuenta los conocimientos y experiencias previas de los estudiantes; en pocos casos se implementan acciones relacionadas con la educación experiencial.

Yo trabajo en el aula de clases empleando lecturas, talleres, trabajos en las que plantean ideas de negocio. (respuestas al cuestionario del docente 4)

Yo utilizo los talleres, análisis de caso, lecturas dirigidas, sustentaciones. (respuestas al cuestionario del docente 8)

Para el desarrollo de la asignatura, las estrategias de enseñanza - aprendizaje básicamente son: inicio de clase con una fase afectiva o de motivación hacia la temática que se va a desarrollar; posteriormente, una fase cognitiva donde se explica y se imparte el conocimiento, y una fase expresiva donde se escenifica el "que hacer" del estudiante. Posteriormente se realizan, clase a clase, entregas parciales de los avances del estudio de mercado. Así, tema enseñado, tema evaluado a través de entregas, que se regresan para realizar las respectivas correcciones y posteriormente pasar a diapositivas para la presentación final del estudio de mercado de la idea de negocio. Aclaro que aparte de las entregas realizo primer y segundo parcial de manera escrita para evaluar conocimientos y el final lo constituye la presentación de todas las entregas trabajadas durante el semestre como un producto final. (respuestas al cuestionario del docente 11)

\section{Conclusiones y recomendaciones}

De acuerdo con la información sistematizada y las categorías obtenidas, al tener en cuenta las declaraciones de los docentes, el emprendimiento se concibe como un conocimiento y como una actitud, para lo cual, la enseñanza de este se desarrolla a través del saber; su alcance llega solo hasta la orientación con los alumnos, para la formulación y evaluación del proyecto emprendedor. Desde este saber, las asignaturas y sus contenidos guardan relación con las temáticas que se considera que se deben abordar a nivel nacional e internacional.

En la categoría de actitudes se encontró que las creencias y percepciones son positivas y favorables frente al tema en cuestión, lo cual puede ser apropiado para la institución en tanto que favorece el desarrollo de iniciativas y proyectos institucionales. En el grupo de docentes se identificó la necesidad de desarrollar emprendimientos que involucren prácticas fuera del aula.

Relacionado con las praxis de los docentes para la enseñanza del emprendimiento se encuentra que en esta institución se adoptan formas que se alejan de tendencias, modelos que han evidenciado funcionar y recomendaciones de autores reconocidos. Este hallazgo representa una oportunidad, puesto que se acoge la apreciación en la que coinciden Bliemel (2014), Crissien (2011) y Varela (2011), por la cual los programas de educación emprendedora se deben adecuar a su contexto.

Para finalizar, se recomienda integrar al eje emprendedor las competencias personales a las que 
hacen referencia la OCDE (2016), Varela (2008; 2011), Mindt y Rieckmann (2017) en una asignatura relacionada con el proyecto de vida, así como generar nuevas investigaciones con las cuales se profundice en las formas de desarrollar la educación emprendedora de los docentes de Unicatólica.

\section{Referencias}

Bliemel, M. (2014). Getting Entrepreneurship Education Out of the Classroom and Into Students' Heads. Entrepreneurship Research Journal, 4(2), 237-260. http://dx.doi.org/10.1515/erj-2013-0053

Cáceres, P. (2003). Análisis cualitativo de contenido: Una alternativa metodológica alcanzable. Psicoperspectivas Revista de la Escuela de Psicología, 2(1), 53-82. http://dx.doi.org/10.5027/ psicoperspectivas-vol2-issue1-fulltext-3

Crissien, J. O. (2011). Enseñando entrepreneurship creación de empresas -estrategias pedagógicas para despertar el espíritu empresarial. Revista Escuela de Administración de Negocios, 71, 100-125.

Cubillos, M. C., y Rodríguez, S. M. (2013). Una mirada a la docencia del emprendimiento en IES colombianas. En M. Benegas (ed.), Formando comunidades para el emprendimiento sustentable: VII WorkShop (pp. 293-312). Corporación Universitaria Remington.

López, F. (2002). El análisis de contenido como método de investigación. Revista de educación, 4, 167-179.

Mejía, J. (2000). El muestreo en la investigación cualitativa. Investigaciones sociales, 4(5), 165-180.

Mindt, L., y Rieckmann, M. (2017). Developing competencies for sustainability driven entrepreneurship in higher education: a literature review of teaching and learning methods. Teoría de la Educación, 29(1), 129-159.
Núñez, L., y Núñez, M. (2018). Papel del profesor motivado en la educación emprendedora en España. Revista empresa y humanismo, 21(1), 7-40 http://dx.doi. org/10.15581/015.XXI.1.7-40

Organización para la Cooperación y el Desarrollo Económicos (OCDE). (2016). Perspectivas económicas de América Latina 2017: Juventud, competencias y emprendimiento. OCDE. http://dx.doi. org/10.1787/leo-2017-es

Pizarro, A. (2000). El análisis de estudios cualitativo. Atención Primaria 25(7), 42-46.

Quintana, A. (2006). Metodología de la investigación científica cualitativa. En A. Quintana y W. Montgomery (eds.), Psicología: Tópicos de actualidad (pp. 47-84). UNMSM.

Robinson, S., Neergaard, H., Tanggaard, L., y Krueger, N. (2016). New Horizons in Entrepreneurship: From Teacher-led to Student-centered Learning. Education + Training, 58(7/8), 661-683. https:// doi.org/10.1108/ET-03-2016-0048

Strategyzer. (2020). Business Model Canvas. https:// wuww.strategyzer.com/canvas

Varela, R. (2008). Innovación empresarial. Arte y ciencia en la creación de empresas. Prentice Hall.

Varela, R. (2011). Educación empresarial basada en competencias empresariales. En Desarrollo, Innovación y Cultura Empresarial vol. 2 Educación Empresarial (pp. 78-119). Universidad Icesi, Centro de Desarrollo del Espíritu Empresarial.

Traverso, G., y Paniagua, K. (2015, junio). La enseñanza del emprendimiento como proceso de diseño. Cuadernos del centro de investigación en economía creativa (CIEC) Centro. (25), 3-18. https://wurus.centro.edu.mx/PDF/ CIEC/cuadernos/CuadernoCIEC_25_emprendimiento_proceso_diseno.pdf 